\title{
Neurotransmitter testing of the urine: a comprehensive analysis
}

Marty Hinz'

Alvin Stein ${ }^{2}$

George Trachte 3

Thomas Uncini ${ }^{4}$

'Clinical Research, NeuroResearch Clinics, Inc., Cape Coral, FL, USA;

${ }^{2}$ Stein Orthopedic Associates,

Plantation, FL, USA; ${ }^{3}$ Department of Physiology and Pharmacology, University of Minnesota Medical School, MN, USA; ${ }^{4}$ DBS Labs, Duluth, MI, USA
Correspondence: Marty Hinz 1008 Dolphin Dr, Cape Coral, FL 33904, USA

Tel +l 2186262220

Fax $+\mid 2186261638$

Email marty@hinzmd.com
This article was published in the following Dove Press journal:

Open Access Journal of Urology

6 October 2010

Number of times this article has been viewed

Abstract: This paper analyzes the statistical correlation of urinary serotonin and dopamine data in subjects not suffering from monoamine-secreting tumors such as pheochromocytoma or carcinoid syndrome. Peer-reviewed literature and statistical analyses were searched and monoamine (serotonin and dopamine) assays defined in order to facilitate their proper interpretation. Many research findings in the literature are novel. Baseline assays completed with no monoamine precursors differ from baseline assays performed on a different day in the same subject. There is currently no scientific basis, value, or predictability in obtaining baseline monoamine assays. Urinary assays performed while taking precursors can demonstrate a lack of correlation or unexpected correlations such as inverse relationships. The only valid model for interpretation of urinary monoamine assays is the "three-phase model" which leads to predictability between monoamine assays and precursor administration in varied amounts.

Purpose: This paper reviews the basic science of urinary monoamine assays. Results of statistical analysis correlating baseline and nonbaseline assays are reported and provide valid methods for interpretation of urinary serotonin and dopamine results.

Patients and methods: Key scientific claims promoting the validity of the urinary neurotransmitter testing (UNT) model applications are discussed. Many of these claims were not supported by the scientific literature. Matched-pairs $t$-tests were performed on several groupings. Results of all statistical tests were compared with peer-reviewed literature.

Results: The statistical analysis failed to support the UNT model. Peer-reviewed literature search failed to verify scientific clams made in support of applications of the UNT model in many cases.

Keywords: serotonin, dopamine, urinary neurotransmitter testing

\section{Introduction}

Three applications have evolved with regard to urinary monoamine assays. The first is one of the older applications used in medicine. This is the use of monoamine assays for screening and diagnosing tumors that secrete serotonin or dopamine (herein referred to as the "tumor model"), such as pheochromocytoma (a catecholamine-secreting tumor) and carcinoid syndrome (a serotonin-secreting tumor). ${ }^{1,2}$ The validity of this type of monoamine testing application is well established in the scientific literature.

The second application is the use of monoamine assays for renal organic cation transporter functional status determination (ie, the OCT model). Even though this model is relatively new, having been developed in 2003, this approach and the urinary serotonin and urinary dopamine applications developed according to this model are 
supported by the scientific literature, having been discussed and documented in several articles since February 2009. . $^{3-5}$

The basis for the OCT model requires two or more serial urinary serotonin and dopamine (ie, monoamine) assays while taking varied amino acid precursor daily dosing amounts. The results are then compared in order to determine the change in urinary serotonin and dopamine levels in response to changes in dosing. A urinary serotonin or dopamine value less than 80 or $475 \mu \mathrm{g}$ of monoamine per $\mathrm{g}$ of creatinine, respectively, indicates a Phase II response. A urinary serotonin or dopamine value greater than 80 or $475 \mu \mathrm{g}$ of monoamine per $\mathrm{g}$ of creatinine, respectively, is interpreted as being in Phase I or Phase III. Differentiation of Phase I from Phase III is as follows. If a direct correlation is found between amino acid dosing and urinary assay response, it is referred to as a Phase III response. An inverse correlation is referred to as a Phase I response. ${ }^{3-5}$ Unexpected results with matched-pairs $t$-test analysis revealed no significant difference when comparing baseline monoamine assays with assays performed while taking supplemental amino acid precursors in the same subject.

Peer-reviewed scientific publications discussing urinary serotonin and urinary dopamine phase analysis according to the OCT model were first published in $2009^{3,4}$ and $2010 .^{5}$ These publications outlined the mechanics of the three-phase model in connection with urinary serotonin and urinary dopamine under a novel renal transporter model. This transporter model potentially describes the etiology of the three-phase response of monoamine assays during the administration of varied amino acid precursor daily dosing values. ${ }^{12}$

The third approach defining applications for the use of monoamine assays is the urinary neurotransmitter testing (UNT) model. This paper discusses the UNT model in depth because it is the only model of the three that lacks valid scientific literature discussing the model or supporting the monoamine assay applications that are being promoted.

The goal of this writing is to assess monoamine assay applications statistically and define the validity of monoamine assays in the absence or presence of supplemental amino acid precursors. The premise of the UNT model is that baseline monoamine assays correlate with and are a good predictor of the peripheral and central nervous system neurotransmitter functional status. The basic assumption for this assertion is that serotonin and dopamine cross the blood-brain barrier ${ }^{6-8}$ and are then filtered at the glomerulus and enter the urine without further interaction with the kidneys. ${ }^{6,8}$ This argument is used on the basis of the UNT model to justify the conclusion that monoamine assays, in the presence and absence of serotonin and dopamine amino acid precursors, correlate with central nervous system and peripheral neurotransmitter functional status. It also asserts that baseline testing is the best approach to determine the neurotransmitter functional status of the central and peripheral nervous systems. ${ }^{7,8,10}$

Other conclusions made in support of utilizing monoamine assays under the urinary neurotransmitter testing model are as follows:

- Administration of amino acid precursors directly impacts urinary monoamine levels; therefore, the results of monoamine assays merely need to be interpreted as being either high or low values s, $^{8,911}$

- Baseline testing of urinary monoamines prior to starting supplemental amino acid precursors is required in order to define the amino acid precursor starting dose needed in treatment ${ }^{8-11}$

- Baseline monoamine assays in the absence of supplemental amino acid precursors are required to diagnose and define the serotonin and dopamine imbalance in the central and peripheral nervous systems ${ }^{6,10}$

- Baseline monoamine assays can serve as a reference point to gauge treatment effectiveness after amino acid precursors are started ${ }^{6,11}$

- Baseline monoamine assays can be used to reduce the risk of side effects when amino acid precursor treatment is started. ${ }^{10}$

\section{Materials and methods}

Statistical analysis was performed for each analyzed grouping considered. The statistical analysis involved the matched-pairs $t$-test. After initiation of supplemental amino acid precursor administration or a change in daily dosing levels was maintained constant, a minimum period of seven days without missing one or more doses was required for data to be considered valid. This time period allows the amino acid precursors and the urinary monoamines to achieve equilibrium in order to ensure that valid urinary serotonin and urinary dopamine test results are obtained. A $P$ value $\leq 0.05$ was considered statistically significant. JMP software (SAS Institute, Cary, NC) was used to perform the statistical analysis.

Processing, management, and assay of the urine samples collected for this study were as follows. Urine samples were collected six hours prior to bedtime, with 4 PM being the most frequent collection time point. The samples were stabilized in $6 \mathrm{~N} \mathrm{HCl}$ to preserve urinary dopamine and urinary serotonin. The urine samples were 
collected after a minimum of one week, during which time the patient was taking a specific daily dose of amino acid precursors of serotonin and dopamine. Samples were shipped to DBS Laboratories. Urinary dopamine and serotonin were assayed utilizing commercially available radioimmunoassay kits (3 CAT RIA IB88501 and IB89527; Immuno Biological Laboratories, Inc., Minneapolis, MN). The DBS laboratory is accredited as a high complexity laboratory by Clinical Laboratory Improvement Amendments to perform these assays.

\section{Results}

Two approaches to analyze the validity of the UNT model were undertaken. The first approach was a literature search intended to test claims made in support of applications for monoamine assays under the UNT model. After an exhaustive search, no indepth valid peer-reviewed studies were found documenting the UNT model. In most cases, the claims justifying use of urinary serotonin and urinary dopamine assays according to the UNT model were contrary to the identified scientific literature. The second approach was the statistical analysis of baseline monoamine assays in the presence or absence of supplemental amino acid precursors in order to assess the UNT model critically.

Five significant divergences from the UNT model from the existing scientific literature were identified. Specifically, divergences were noted from the established science, ie, serotonin and dopamine do not cross the blood-brain barrier ${ }^{3,5,12,13}$ and peripheral serotonin and dopamine are filtered at the glomerulus and then enter the proximal tubules. ${ }^{5}$ They are then actively transported into the proximal convoluted renal tubule cells where they are essentially completely metabolized. ${ }^{5}$ Due to the high efficiency of this metabolic process, significant amounts of serotonin and dopamine filtered at the glomerulus do not reach the urine in patients not suffering from a tumor secreting serotonin or dopamine. ${ }^{3,5}$ From a practical standpoint, urinary serotonin and urinary dopamine represent serotonin and dopamine that have not previously been in the central or peripheral nervous system. ${ }^{3,5}$ The literature notes that urinary serotonin and urinary dopamine are monoamines that are newly synthesized from serotonin and dopamine amino acid precursors by the kidneys in the proximal convoluted renal tubule cells. ${ }^{3,5}$ These newly synthesized serotonin and dopamine molecules are then either transported out of the proximal convoluted renal tubule cells across the basolateral membrane and then into the peripheral system via the renal vein or across the apical membrane and then into the urine..$^{3-5,14,15}$ It is noted that there are many other renal interactions that exist between synthesis of serotonin and dopamine transported across the basolateral membrane and the apical membrane prior to arriving at the final destination of the renal vein or urine, respectively. These interactions appear small in comparison with the effects of the basolateral monoamine transporter and the apical monoamine transporter under the three-phase model. ${ }^{5}$ There is also no correlation between urinary serotonin and dopamine levels and the serotonin or dopamine levels within the central and peripheral nervous systems. ${ }^{3-5}$ The renal interaction of urinary serotonin, urinary dopamine, and their amino acid precursors is counterintuitive. It is expected that when serotonin and/or dopamine amino acid precursors are administered, levels of the associated urinary serotonin or urinary dopamine will increase or decrease with increases or decreases in the amino acid precursor daily dosing levels, ie, a direct relationship. The literature reveals that this is not the predominant response. Outcomes are not intuitive because the process is complex, and there is no simple, dominant, direct relationship between serotonin and dopamine amino acid dosing and monoamine assays. Instead, a complex interaction is found, giving rise to the three-phase model, as we have previously proposed..$^{3-5}$ Furthermore, there is no significant statistical difference between baseline monoamine levels in the urine and those resulting from administration of monoamine precursors. Given that support for this is not found in the literature, the following statistical analysis is put forth. The data for the following analysis was obtained from the DBS Laboratories monoamine assay database. The database was assembled according to the criteria discussed in the Materials and methods section.

By definition, the laboratory baseline reference range for a given assay is calculated by taking all baseline data generated for that assay, then defining the group of values that are within two SDs from the mean. This grouping size represents approximately $95 \%$ of the initial group data generated. In the following reports of statistical analysis, when use of the reference range values is referred to, the following values were used. A laboratory promoting the UNT model has defined the urinary serotonin reference range as $150-300 \mu \mathrm{g}$ of serotonin per $\mathrm{g}$ of creatinine. The same laboratory defined the urinary dopamine reference range as $150-300 \mu \mathrm{g}$ of dopamine per $\mathrm{g}$ of creatinine. ${ }^{9}$

\section{Urinary serotonin at baseline versus while taking 5-hydroxytryptophan}

Matched-pairs groupings were queried from the database as follows. Two urinary serotonin samples from the same subject were obtained, one sample while taking no supplemental amino acid precursors and the other sample while taking 
5-hydroxytryptophan (5-HTP), and these were match-paired together. A group of these matched-pairs samples were then defined for analysis, revealing a group of $n=167$. The serotonin reference range values as reported above were used to query the baseline urinary matched-pairs serotonin group of $n=167$ further, revealing a group of $n=103$. The group taking 5-HTP was then queried from the group of $n=103$ using the parameter 5-HTP $<301 \mathrm{mg}$ per day, to give a final matched-pairs group of $\mathrm{n}=78$ for analysis.

The final matched-pairs group was then analyzed using a $t$-test, and a $P$ value of 0.0809 was found, indicating lack of a significant statistical difference between baseline urinary serotonin levels and serotonin levels when taking less than $301 \mathrm{mg}$ of 5-HTP per day.

\section{Urinary dopamine at baseline versus while taking levodopa}

Matched-pairs groups were queried from the database as follows. Two samples from each subject, one sample taking no supplemental amino acid precursors and the other sample taking levodopa, were paired together. This revealed a group of $n=617$. The baseline assay portion of the entire matchedpairs group was queried with the dopamine reference range values reported earlier, to give a population size of $n=230$. The group taking levodopa was then queried to find only subjects taking less than $361 \mathrm{mg}$ of levodopa per day, leading to a final population size of $n=166$. This matched-pairs group was then analyzed using a matched-pairs $t$-test, and a $P$ value of 0.0742 was found, indicating no significant statistical difference between baseline dopamine assays and dopamine assays performed while taking less then $361 \mathrm{mg}$ of levodopa per day.

\section{Baseline serotonin assays from different days in the same subject}

Data were analyzed in the following manner, with the following numbers reported in $\mu \mathrm{g}$ of serotonin per $\mathrm{g}$ of creatinine. From a matched-pairs group of $n=146$, the mean (SD) for both baseline serotonin urinary assay groups was determined. For Group 1, the mean serotonin value was found to be 239.0 ( \pm 2282.8 ). For Group 2 (baseline testing performed on a different day after the first assay) the mean serotonin value was found to be $273.2( \pm 8214.51)$. All data greater than the value found in calculating the sum of two SDs plus the mean were removed from consideration, revealing a group of $n=134$. The matched-pairs grouping was then analyzed using the matched-pairs $t$-test. The baseline urinary serotonin assay grouping analysis revealed a $P$ value of 0.0080 . These findings indicate that baseline urinary levels do differ in a statistically significant manner when baseline assays are performed on different days for the same subject and are not uniform or reproducible from day to day.

\section{Baseline dopamine assays from different days in the same subject}

Data were analyzed in the following manner, with numbers reported in $\mu \mathrm{g}$ of dopamine per $\mathrm{g}$ of creatinine. From a matched-pairs group of $n=146$, the mean SD for both baseline serotonin urinary assay groups was determined. For Group 1, the mean dopamine value was found to be 144.0 $( \pm 286.9)$. For Group 2 (baseline testing performed on a different day after the first assay), the mean dopamine value was found to be $198.6( \pm 484.8)$. All data greater than the value found in calculating the sum of two SD plus the mean were removed from consideration, revealing a group of $n=138$. The matched-pairs grouping was then analyzed using the matched-pairs $t$-test. The baseline urinary serotonin assay grouping analysis revealed a $P$ value of 0.0049 . These findings indicate that baseline urinary dopamine levels do differ in a statistically significant manner when baseline assays are performed on different days in the same subject, and are not uniform or reproducible from day to day.

\section{Discussion}

The focus of this research is the applications of urinary serotonin and dopamine assays, whereby three distinctly different application models of monoamine assays are being promoted. The basis of the tumor model is screening for a monoaminesecreting tumor. This methodology is well founded. The OCT model is a relatively new application of monoamine assays, but its validity is supported by the literature..$^{3-5}$ The third application model for monoamine assays, the urinary neurotransmitter testing model, has no indepth, valid, peerreviewed scientific literature to support its use. The UNT model distinguishes itself from the two other approaches by requiring use of baseline urinary monoamine assays, and advocates a direct relationship between urinary serotonin and urinary dopamine when the serotonin and dopamine amino acid precursor daily dosing levels are varied. The following is a consolidation of the findings and scientific concepts discussed in this paper with the claims and approach for use of monoamine assay applications under the UNT model.

Significant challenges to the urinary neurotransmitter testing model include the widely recognized finding that serotonin and dopamine do not cross the blood-brain barrier. ${ }^{16-19}$ 
In support of applications for urinary serotonin and urinary dopamine assays, the UNT model claims that serotonin and dopamine do cross the blood-brain barrier. ${ }^{6-8}$ This assertion is widely known to be untrue. ${ }^{16-19}$

No significant amount of serotonin and dopamine filtered at the glomerulus reaches the urine. Serotonin and dopamine found in the urine are newly synthesized in the kidneys, and their levels are a function of the interaction between the basolateral monoamine transporters and the apical monoamine transporters of the proximal convoluted renal tubule cells. ${ }^{19}$ The UNT model claims that serotonin and dopamine are merely filtered at the glomerulus, and then enter the urine without further renal interactions. ${ }^{6}$ This assertion is not supported by review of the relevant science.

Urinary serotonin and urinary dopamine found in the urine have no correlation with brain or peripheral serotonin and dopamine levels. Significant levels of urinary serotonin and urinary dopamine molecules assayed in the urine have never been shown in the brain or peripheral nervous system. ${ }^{3,5}$ The UNT model, based on assertions that serotonin and dopamine cross the blood-brain barrier and are then simply filtered at the glomerulus and enter the urine, claims that urinary monoamine assays represent the functional neurotransmitter status of the central nervous system, peripheral nervous system, and urine. ${ }^{1}$ This assertion again is not supported by the relevant science.

There is no consistent direct relationship between serotonin and dopamine amino acid precursor daily dosing levels and the amount of serotonin and dopamine that appears in the urine on monoamine assays. ${ }^{3-5}$ The peer-reviewed literature notes that there is no relationship between administration of the serotonin precursor, 5-HTP, in varied doses and subsequent urinary serotonin levels. ${ }^{4}$ The literature also notes that there is a correlation between administration of L-tyrosine and urinary dopamine levels, but this is an inverse relationship, ${ }^{4}$ and not the direct relationship predicted by the UNT model. ${ }^{6,7}$ The UNT model advocates that there is a dominant direct correlation between amino acid doses and urinary serotonin and urinary dopamine found on assay. ${ }^{6,7}$ This leads to the assertion under the UNT model that simply determining whether the urinary serotonin and urinary dopamine levels found on assay are high or low is the focal point of proper monoamine assay interpretation. ${ }^{6,7}$ This assertion is not supported on review of the science involved.

Statistical analysis of baseline monoamine assays reveals that these assays do not predict the response to precursor therapy. They differ significantly with subsequent baseline assays undertaken on different days from the same subject, and no significant difference exists with assays performed when amino acid precursors are taken. These findings are contrary to the assertions of the UNT model..$^{6-8,11}$

The UNT model claims that baseline monoamine assays obtained prior to ingestion of supplemental amino acid precursors can identify neurotransmitter imbalance in the central nervous system, peripheral nervous system, and urine. ${ }^{6-8}$ Due to the statistical difference in baseline monoamine assays in the same subject from day to day, an unlimited number of different neurotransmitter imbalances might theoretically be diagnosed with serial assays performed on many different days from the same subject. There is a statistical difference between baseline urinary serotonin and urinary dopamine assays in subjects not harboring a monoaminesecreting tumor. The assertion that baseline monoamine assays can diagnose central nervous system, peripheral nervous system, and urinary neurotransmitter dysfunction is not supported on review of the scientific literature.

The UNT model also claims that baseline assays of urinary serotonin and urinary dopamine are required prior to starting serotonin and/or dopamine amino acid precursors to assist in selecting the optimal daily serotonin and dopamine amino acid precursor doses. ${ }^{8-10}$ Using any laboratory criteria to diagnose serotonin and dopamine imbalance prior to selecting the starting point of amino acid dosing gives results that differ statistically from day to day and are not reproducible. The assertion on the part of the UNT model that baseline monoamine assays are needed to determine a starting point for serotonin and dopamine amino acid precursor treatment is not supported.

The UNT model claims that baseline assays are required to minimize side effects when treatment with amino acid precursors is started. The results of baseline assays obtained from the same subject on different days vary statistically, and are not reproducible relative to the first baseline assay obtained. The ability to minimize side effects claimed on the basis of the UNT model is not supported by the reported science.

The UNT model incorrectly asserts that baseline monoamine assays can serve as a reference point during treatment to gauge effectiveness of treatment when serotonin and dopamine amino acid precursors are started. ${ }^{8,10}$ As noted already, there is a significant statistical difference between values found with baseline monoamine assays and baseline assays performed on a different day in the same subject, leading to a host of different reference points being generated when baseline assays are obtained on multiple days. The baseline assays cannot be used as a reference point to measure treatment progress or indicate results of treatment. 
The only valid correlation that exists between monoamine assays performed with and without administration of amino acid precursors in subjects not suffering from a monoaminesecreting tumor is the three-phase model described in the literature. When the three-phase model is applied correctly to urinary serotonin and urinary dopamine assay results, it leads to a predictable course of outcomes with urinary serotonin and urinary dopamine assay interpretation. The three-phase model is based on the interaction between the newly synthesized serotonin and dopamine by the kidneys with the basolateral monoamine (serotonin and dopamine) transporters and the apical monoamine (serotonin and dopamine) transporters of the proximal convoluted renal tubule cells of the kidneys, leading to the serotonin and dopamine that is found in the urine on assay. ${ }^{3-5}$

\section{Conclusion}

The application and interpretation of baseline monoamine assays according to the urinary neurotransmitter testing model is not a valid approach because there is a significant statistical difference between baseline monoamine assays and monoamine assays obtained on a different day from the same subject and no significant statistical difference in subsequent monoamine assays performed while taking amino acid precursors. The UNT model has no ability to diagnose central or peripheral nervous system serotonin and dopamine imbalance using baseline monoamine assays in subjects not suffering from monoamine-secreting tumors. Urinary serotonin and urinary dopamine assays are not assays of serotonin and dopamine that have been in the central nervous system. Serotonin and dopamine do not cross the blood-brain barrier. Significant amounts of urinary serotonin and urinary dopamine found on assay have not been in the brain or in the peripheral system. Urinary serotonin and urinary dopamine are filtered at the glomerulus and are then metabolized in the kidneys, with no significant amounts of serotonin or dopamine filtered at the glomerulus being found in the urine. Levels of urinary serotonin and urinary dopamine found on assay are newly synthesized in the kidneys, and are a function of the interaction between the basolateral monoamine transporters and apical monoamine transporters of the proximal convoluted renal tubule cells.

A simple direct relationship between the daily dosing levels of amino acid precursors and monoamine assays does not exist in most cases. Due to complex renal physiologic interactions between serotonin and dopamine newly synthesized by the kidneys, a complex relationship is observed that is defined by the three-phase model described in the already published peer-reviewed literature.

The goal of this paper is to spark interest, research, awareness, and scrutiny of the topics discussed. A laboratory assay is only valid if properly interpreted. Correct interpretation of monoamine assays while taking amino acid precursors is complex, and not a direct linear relationship as predicted by the UNT model.

\section{Disclosure}

TU and $\mathrm{MH}$ are director and owner of DBS Laboratories, Duluth, Minnesota respectively. AS and GT have no conflicts of interest to report in this work.

\section{References}

1. Oates JA, Sjoerdsma A. A unique syndrome associated with secretion of 5-hydroxytryptophan by metastatic gastric carcinoids. Am J Med. 1962;32:333-342.

2. Szakacs JE, Cannon AL. Noreprinephrine myocarditis. Am J Clin Pathol. 1958;30:425-434.

3. Hinz M. Depression. In: Kohlstadt I, editor. Food and Nutrients in Disease Management. CRC Press; 2009.

4. Trachte G, Uncini T, Hinz M. Both stimulatory and inhibitory effects of dietary 5-hydroxytryptophan and tyrosine are found on urinary excretion of serotonin and dopamine in a large human population. Neuropsychiatr Dis Treat. 2009;5:227-235.

5. Hinz M, Stein A, Uncini T. The dual gate lumen model of renal monoamine transport. Neuropsychiatr Dis Treat. 2010;6:387-392.

6. Alts J, Alts D, Bull M. Urinary Neurotransmitter Testing: Myths and Misconceptions. Osceola, WI: NeuroScience, Inc.; 2007.

7. Watkins R. Validity of urinary neurotransmitter testing with clinical applications of CSM (Communication System Management) model. Asheville, NC: Sanesco International; 2009. Available at: http://www.neurolaboratory.net/lab/neurolab\%20pdf\%20files/2009\%20 Urinary $\% 20$ NT\%20White\%20Paper.pdf. Accessed 2010 Aug 4.

8. Theirl S. Clinical relevance of neurotransmitter testing. The Original Internist. Dec 2009. Available at: http://www.clintpublication.com/ documents/Dec_OI_2009.pdf. Accessed 2010 Aug 4.

9. Sanesco. Neurolab baseline sample report. Available at: http://sanesco.net/images/files/resourcelibrary/baseline_sample_report. pdf Accessed 2010 Jul 2.

10. Neuroscience. Assessing nutritional imbalances. Available at: https://www.neurorelief.com/index.php?option=com_content\&task=view\& id=131\&Itemid=48. Accessed 2010 Jul 2.

11. Kellermann G, Bull M, Ailts J, et al. Understanding diurnal variation. Technical Bulletin Issue 4. Osceola, WI: NeuroScience, Inc.; 2004: Jan 9. Available at: https://www.neurorelief.com/index.php?option = com_co ntent\&task=view\&id=224\&Itemid=48. Accessed $2010 \mathrm{Jul} 2$.

12. Carley C, Radulovacki M. Role of peripheral serotonin in the regulation of central sleep apneas in rats. Chest. 1999;115:1397-1401.

13. Volkow N, Fowler JS, Gatley J, et al. PET evaluation of the dopamine system of the human brain. J Nucl Med. 1996;37: 1242-1256.

14. Wang Y, Berndt T, Gross T, Peterson M, So M, Know F. Effect of inhibition of MAO and COMT on intrarenal dopamine and serotonin and on renal function. Am J Physiol Regul Integr Comp Physiol. 2001;280:R248-R254.

15. Vieira-Coelho MA, Soares-Da-Silva P. Apical and basal uptake of L-dopa and L-5-HTP and their corresponding amines, dopamine and 5-HT, in OK cells. Am J Physiol. 1997;272(5 Pt 2):F632-F639. 
16. Pyle AC, Argyropoulos SV, Nutt DJ. The role of serotonin in panic: Evidence from tryptophan depletion studies. Acta Neuropsychiatr. 2004;16:79-84.

17. Verde G, Oppizzi G, Colussi G, et al. Effect of dopamine infusion on plasma levels of growth hormone in normal subjects and in agromegalic patients. Clin Endocrinol (Oxf). 1976;5:419-423.
18. Gozzi A, Ceolin L, Schwarz A, et al. A multimodality investigation of cerebral hemodynamics and autoregulation in pharmacological MRI. Magn Reson Imaging. 2007;25:826-833.

19. Ziegler MG, Aung M, Kennedy B. Sources of human urinary epinephrine. Kidney Int. 1997;51:324-327.

Open Access Journal of Urology

\section{Publish your work in this journal}

The Open Access Journal of Urology is an international, peer-reviewed, open access journal publishing original research, reports, editorials, reviews and commentaries on all aspects of adult and pediatric urology in the clinic and laboratory including the following topics: Pathology, pathophysiology of urological disease; Investigation and treatment of
Dovepress

urological disease; Pharmacology of drugs used for the treatment of urological disease. The manuscript management system is completely online and includes a very quick and fair peer-review system, which is all easy to use. Visit http://www.dovepress.com/testimonials.php to read real quotes from published authors.

Submit your manuscript here: http://www.dovepress.com/open-access-journal-of-urology-journal 